\title{
A NOTE ON AN ORDER BETWEEN OBJECT-ORIENTED SOFT CONCEPTS IN A SOFT CONTEXT
}

\author{
Won Keun Min \\ Department of Mathematics, Kangwon National University, Chuncheon 24341, Korea. \\ ORCID: 0000-0002-3439-2255
}

\begin{abstract}
:
The purpose of this work is to study the algebraic structure in the family of all the $m$-concepts, so we introduce the notion of an order in the set of all $m$-concepts and show that the ordered set is a complete lattice. And, we discover what is the condition for the isomorphic relation between two $m$-concept lattices in a given soft context.
\end{abstract}

\section{INTRODUCTION}

Formal concept analysis [10] was introduced by Wille, which is an important theory for the research of information structures induced by a binary relation between the set of attributes and objects attributes. The three basic notions of FCA are formal context, formal concept, and concept lattice. A formal context is a kind of information system, which is a tabular form of an object-attribute value relationship [2, 3, 9]. A formal concept is a pair of a set of objects as called the extent and a set of attributes as called the intent. The set of all formal concepts together with the order relation forms a complete lattice called the concept lattice $[9,10]$. In order to deal complicated problems, Molodtsov introduced the concept of soft set in [8]. The operations for the soft set theory was introduced by Maji et al. in [4]. In [1], Ali et al. proposed new operations modified some concepts introduced by Maji. We have formed a soft context by combining the concepts of the formal context and the soft set defined by the set-valued mapping in [6]. Additionally, we introduced and studied the new concepts named soft concepts and soft concepts lattices.

In [11], Yao introduced a new concept called an object oriented formal concept in a formal context by using the notion of approximation operations.

And also, by using the two operation, we investigated the new concept of $m$-concepts related closely the object oriented concept in formal context in [7].

In this paper, we introduce the notion of an order in the set of all $m$-concepts and show that the ordered set is a complete lattice. And, we discover what is the condition for the isomorphic relation between two $m$-concept lattices in a given soft context.

\section{PRELIMINARIES}

A formal context is a triplet $(U, V, I)$, where $U$ is a non-empty finite set of objects, $V$ is a nonempty finite set of attributes, and $I$ is a relation between $U$ and $V$. Let $(U, V, I)$ be a formal context. For a pair of elements $x \in U$ and $y \in V$, if $(x, y) \in I$, then it means that object $x$ has attribute $y$ and we write $x I y$. The set of all attributes with a given object $x \in U$ and the set of all objects with a a given attribute $y \in V$ are denoted as the following $[9,10]$ :

$$
x^{*}=\{y \in V \mid x I y\} ; y^{*}=\{x \in U \mid x I y\} .
$$

And, the operations for the subsets $X \subseteq U$ and $Y \subseteq V$ are defined as:

$$
X^{*}=\{y \in V \mid \text { for all } x \in X, x I y\} ; \quad Y^{*}=\{x \in
$$
$U \mid$ for all $y \in Y, x I y\}$.

In a formal context $(U, V, I)$, a pair $(X, Y)$ of two sets $X \subseteq U$ and $Y \subseteq V$ is called a formal concept of $(U, V, I)$ if $X=Y^{*}$ and $B=Y^{*}$, where $X$ and $Y$ are called the extent and the intent of the formal concept, respectively.

Let $U$ be a universe set and $E$ be a collection of properties of objects in $U$. We will call $E$ the set of parameters with respect to $U$.

A pair $(F, E)$ is called a soft set [8] over $U$ if $F$ is a set-valued mapping of $E$ into the set $P(U)$ of all subsets of the set $U$, i.e.,

$$
F: E \rightarrow P(U)
$$

In other words, for $a \in E$, every set $F(a)$ may be considered as the set of $a$-elements of the soft set $(F, E)$.

Let $U=\left\{z_{1}, z_{2}, \ldots, z_{m}\right\}$ be a non-empty finite set of objects, $E=\left\{e_{1}, e_{2}, \ldots, e_{n}\right\}$ a non-empty finite set of attributes, and $F: E \rightarrow P(U)$ a soft set. Then the triple $(U, E, F)$ is called a soft context [6].

And, in a soft context $(U, E, F)$, we introduced the following mappings:

For each $Z \in P(U)$ and $Y \in P(E)$,

(1) $\mathbf{F}^{+}: P(E) \rightarrow P(U)$ is a mapping defined as $\mathbf{F}^{+}(Y)=$ $\bigcap_{y \in Y} F(y)$;

(2) $\mathbf{F}^{-}: P(U) \rightarrow P(E)$ is a mapping defined as $\mathbf{F}^{-}(Z)=$ $\{a \in E: Z \subseteq F(a)\}$;

(3) $\Psi: P(U) \rightarrow P(U)$ is an operation defined as $\Psi(Z)=$ $\mathbf{F}^{+} \mathbf{F}^{-}(Z)$.

Then $Z$ is called a soft concept [6] in $(U, E, F)$ if $\Psi(Z)=$ $\mathbf{F}^{+} \mathbf{F}^{-}(Z)=Z$. The set of all soft concepts is denoted by $s C(U, E, F)$.

In [7], we introduced the notion of $m$-concepts which is independent of the notion of soft concepts to each other as the following: For each $X \in P(U)$,

For each $Z \in P(U)$ and $Y \in P(E)$,

(1) $\mathbb{F}: P(A) \rightarrow P(U)$ is a mapping defined as $\mathbb{F}(C)=$ $\cup_{c \in C} F(c)$; 
$(2) \overleftarrow{\mathbb{F}}: P(U) \rightarrow P(A)$ is a mapping defined as $\overleftarrow{\mathbb{F}}(X)=$ $\{c \in A: F(c) \subseteq X\}$

Theorem 2.1 ([7]). Let $(U, A, F)$ be a soft context, $S, T \subseteq U$ and $B, C \subseteq A$. Then we have:

(1) If $S \subseteq T$, then $\overleftarrow{\mathbb{F}}(S) \subseteq \overleftarrow{\mathbb{F}}(T)$; if $B \subseteq C$, then $\mathbb{F}(B) \subseteq \mathbb{F}(C)$;

(2) $\mathbb{F} \overleftarrow{\mathbb{F}}(S) \subseteq S ; \overleftarrow{\mathbb{F}} \mathbb{F}(B) \subseteq B$

(3) $\overleftarrow{\mathbb{F}}(S \cap T)=\overleftarrow{\mathbb{F}}(S) \cap \overleftarrow{\mathbb{F}}(T), \mathbb{F}(B \cup C)=\mathbb{F}(B) \cup \mathbb{F}(C)$;

(4) $\overleftarrow{\mathbb{F}}(S)=\overleftarrow{\mathbb{F}} \mathbb{F} \overleftarrow{\mathbb{F}}(S), \mathbb{F}(B)=\mathbb{F} \overleftarrow{\mathbb{F}} \mathbb{F}(B)$

Let $\Phi: P(U) \rightarrow P(U)$ be an operation defined by $\Phi(X)=$ $\mathbb{F} \overleftarrow{\mathbb{F}}(X)$ for $X \in P(U)$.

Then for $X \in P(U), X$ is called an $m$-concept (or object oriented soft concept $)[7]$ in $(U, A, F)$ if $\Phi(X)=\mathbb{F} \overleftarrow{\mathbb{F}}(X)=X$. The set of all $m$-concepts is denoted by $m(U, A, F)$.

\section{MAIN RESULTS}

First, for a soft context $(U, A, F)$ and $C \subseteq A$, we consider a set-valued mapping $F_{C}: C \rightarrow P(U)$ defined by $F_{C}(c)=F(c)$ for all $c \in C$. Then the set-valued mapping $F_{C}$ induces a soft set $\left(F_{C}, C\right)$ and a soft context $\left(U, C, F_{C}\right)$. Then we consider the operations $\mathbb{F}_{C}, \overleftarrow{\mathbb{F}_{C}}, \Phi_{C}$ as the following:

$\mathbb{F}_{C}: P(C) \rightarrow P(U)$ is a mapping defined by $\mathbb{F}_{C}(B)=$ $\cup_{b \in B} F_{C}(b)$ for each $B \in P(C)$.

$\overleftarrow{\mathbb{F}_{C}}: P(U) \rightarrow P(C)$ is a mapping defined by $\overleftarrow{\mathbb{F}_{C}}(X)=$ $\left\{c \in C: F_{C}(c) \subseteq X\right\}$ for each $X \in P(U)$.

An associated operation $\Phi_{C}: P(U) \rightarrow P(U)$ is also well defined by for each $X \in P(U), \Phi_{C}(X)=\mathbb{F}_{C} \overleftarrow{\mathbb{F}_{C}}(X)$.

Lemma 3.1. Let $(U, A, F)$ be a soft context, $C \subseteq A$ and $X \subsetneq U$. Then

(1) $\mathbb{F}_{C}(X) \subseteq \overleftarrow{\mathbb{F}}(X)$

(2) $\overleftarrow{\mathbb{F}_{C}}(X)=\overleftarrow{\mathbb{F}}(X) \cap C$

Proof. Obvious.

Theorem 3.2. Let $(U, A, F)$ be a soft context, $X, Y \subseteq U$ and $B, C, E \subseteq A$. Then we have the following things:

(1) If $X \subseteq Y$, then $\overleftarrow{\mathbb{F}_{C}}(X) \subseteq \overleftarrow{\mathbb{F}_{C}}(Y)$; if $B \subseteq E$, then $\mathbb{F}_{C}(B) \subseteq \mathbb{F}_{C}(E)$

(2) $\mathbb{F}_{C} \overleftarrow{\mathbb{F}_{C}}(X) \subseteq X ; \overleftarrow{F}_{C} \mathbb{F}_{C}(B) \subseteq B$;

(3) $\overleftarrow{\mathbb{F}_{C}}(X \cap Y)=\overleftarrow{\mathbb{F}_{C}}(X) \cap \overleftarrow{\mathbb{F}_{C}}(Y), \mathbb{F}_{C}(B \cup E)=\mathbb{F}_{C}(B) \cup$ $\mathbb{F}_{C}(E)$;

(4) $\overleftarrow{\mathbb{F}_{C}}(X)=\overleftarrow{\mathbb{F}_{C}} \mathbb{F}_{C} \overleftarrow{\mathbb{F}_{C}}(X), \mathbb{F}_{C}(B)=\mathbb{F}_{C} \overleftarrow{\mathbb{F}_{C}} \mathbb{F}_{C}(B)$

Proof. It is obvious from the notions of $\mathbb{F}_{C}, \overleftarrow{\mathbb{F}_{C}}$ and $\Phi_{C}$.

Let $(U, A, F)$ be a soft context, $X \in P(U)$ and $C \subseteq A$. Then $X$ is called $m$-concept in $\left(U, C, F_{C}\right)$ if $\Phi_{C}(X)=$ $\mathbb{F}_{C} \overleftarrow{\mathbb{F}} C(X)=X$. The set of all $m$-concepts will be denoted by $m\left(U, C, F_{C}\right)$.

Theorem 3.3 ([7]). Let $(U, A, F)$ be a soft context. Then we have:

(1) $\Phi(\emptyset)=\emptyset$.

(2) $\Phi(X)$ is an $m$-concept.

(3) For $B \subseteq A, \mathbb{F}(B)$ is an $m$-concept.
(4) For $a \in A, F(a)$ is an $m$-concept.

(5) $X$ is an $m$-concept if and only if there is some $B \subseteq A$ such that $X=\mathbb{F}(B)$.

By Theorem 3.3, the next theorem is obviously obtained:

Theorem 3.4. Let $(U, A, F)$ be a soft context, $X \subseteq U$ and $B, C \subseteq A$. Then

(1) $\Phi_{C}(\emptyset)=\emptyset$.

(2) $\Phi_{C}(X)$ is an $m$-concept in $\left(U, C, F_{C}\right)$.

(3) For each $B \subseteq C, \mathbb{F}_{C}(B)$ is an $m$-concept in $\left(U, C, F_{C}\right)$.

(4) For each $c \in C, F(c)$ is an $m$-concept in $\left(U, C, F_{C}\right)$.

(5) $X$ is an $m$-concept in $\left(U, C, F_{C}\right)$ if and only if $X=$ $\mathbb{F}_{C}(B)$ for some $B \in P(C)$.

Theorem 3.5. Let $(U, A, F)$ be a soft context and $C \subseteq A$. Then

(1) $m\left(U, C, F_{C}\right)=\operatorname{Im}\left(\mathbb{F}_{C}\right)$.

(2) If $\mathbb{F}_{C}\left(B_{1}\right), \cdots, \mathbb{F}_{C}\left(B_{n}\right) \in \operatorname{Im}\left(\mathbb{F}_{C}\right)$, then $\mathbb{F}_{C}\left(B_{1}\right) \cup \cdots \cup$ $\mathbb{F}_{C}\left(B_{n}\right) \in \mathbf{I m}\left(\mathbb{F}_{C}\right)$.

Proof. (1) By (3) of Theorem 3.4, it is easily obtained.

(2) For $B_{1} \cdots B_{n} \in P(C)$, by (3) of Theorem 3.2, $\mathbb{F}_{C}\left(B_{1}\right) \cup$ $\cdots \cup \mathbb{F}_{C}\left(B_{n}\right)=\mathbb{F}_{C}\left(B_{1} \cup \cdots \cup B_{n}\right)$. Since $B_{1} \cup \cdots \cup$ $B_{n} \in P(C)$, by (3) of Theorem 3.4, the statement (2) is obtained.

Theorem 3.6. Let $(U, A, F)$ be a soft context and $\mathcal{S}_{C}=$ $\left\{F_{C}(c) \mid c \in C \subseteq A\right\}$ for the soft set $\left(F_{C}, C\right)$. Then

(1) $\mathcal{S}_{C} \subseteq m\left(U, C, F_{C}\right)$ :

(2) For each $X \in m\left(U, C, F_{C}\right)$, there is $S_{1}, S_{2}, \cdots, S_{n} \in \mathcal{S}_{C}$ satisfying $X=\cup S_{i}, i=1,2, \cdots, n$.

Proof. (1) By (4) of Theorem 3.4, it is obvious.

(2) By (4) of Theorem 3.4, there is a $B \in P(C)$ satisfying $X=\mathbb{F}_{C}(B)$. So, $X=\mathbb{F}_{C}(B)=\cup_{b \in B} F_{C}(b)$ and $F_{C}(b) \in \mathcal{S}_{C}$.

Theorem 3.7. Let $(U, A, F)$ be a soft context. Then for $C \subseteq A, m\left(U, C, F_{C}\right) \subseteq m(U, A, F)$.

Proof. For $X \in m\left(U, C, F_{C}\right)$, by Theorem 3.4, there is $B \in P(C)$ satisfying $X=\mathbb{F}_{C}(B)$. From Lemma 3.1, $X=$ $\mathbb{F}_{C}(B)=\mathbb{F}(B)$ for $B \in P(C)$. From $m(U, A, F)=\operatorname{Im}(\mathbb{F})$ in [7], it implies $X \in m(U, A, F)$. So, $m\left(U, C, F_{C}\right) \subseteq$ $m(U, A, F)$.

Now, we define an order between two $m$-soft concepts in $m(U, A, F)$ as the following:

Definition 3.8. Let $(U, A, F)$ be a soft context and $X, Y \in$ $m(U, A, F)$.

$$
X \preceq Y \text { if and only if } X \subseteq Y \text {. }
$$

$X$ is called a sub-m-concept of $Y$, and $Y$ is called a super$m$-concept of $X$. For the ordered set $(m(U, A, F), \preceq)$, the infimum $\wedge$ and supremum $\vee$ are defined by:

$$
X \wedge Y=\Phi(X \cap Y) ; \quad X \vee Y=X \cup Y .
$$

Example 3.9. For $U=\{1,2,3,4,5\}, A=\{a, b, c, d, e\}$, Let us consider a soft context $(U, A, F)$ as shown in Table 1 . 
International Journal of Engineering Research and Technology. ISSN 0974-3154 Vol.13, No.3 (2020), pp. 548-551

(c) International Research Publication House. https://dx.doi.org/10.37624/IJERT/13.3.2020.548-551

Table 1:A formal context

\begin{tabular}{|c|c|c|c|c|c|}
\hline- & $\mathrm{a}$ & $\mathrm{b}$ & $\mathrm{c}$ & $\mathrm{d}$ & $\mathrm{e}$ \\
\hline 1 & 1 & 1 & 0 & 1 & 1 \\
2 & 1 & 0 & 1 & 1 & 1 \\
3 & 0 & 1 & 0 & 1 & 0 \\
4 & 0 & 0 & 0 & 0 & 0 \\
5 & 0 & 0 & 1 & 0 & 1 \\
\hline
\end{tabular}

Then, $(F, A)$ is a soft set as follows:

$$
\begin{gathered}
F(a)=\{1,2\} ; \quad F(b)=\{1,3\} ; \quad F(c)=\{2,5\} ; \\
F(d)=\{1,2,3\} ; \quad F(e)=\{1,2,5\} .
\end{gathered}
$$

For the soft context $(U, A, F)$,

$m(U, A, F)=\operatorname{Im}(\mathbb{F})$

$=\left\{\mathbb{F}(C) \mid \mathbb{F}(C)=\cup_{c \in C} F(c)\right.$ for $\left.C \in P(A)\right\}$

$=\{\emptyset,\{1,2\},\{1,3\},\{2,5\},\{1,2,3\},\{1,2,5\},\{1,2,3,5\}, U\}$.

Hence, $m L(U, A, F)$ is obtained as shown in the below diagram:

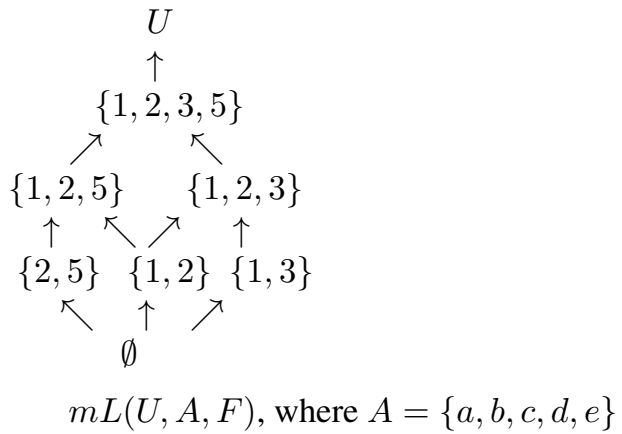

Theorem 3.10. Let $(U, A, F)$ be a soft context. Then $(m(U, A, F), \preceq, \wedge, \vee)$ is complete lattice.

Proof. (1) Let $X, Y \in m(U, A, F)$. Then from Theorem 3.4, there exist $B, C \in P(A)$ such that $\mathbb{F}(B)=X$ and $\mathbb{F}(C)=Y$. By Theorem 3.2, $\mathbb{F}(B) \cup \mathbb{F}(C)=\mathbb{F}(B \cup C)$ and $X \cup Y=\mathbb{F}(B \cup C)$. It implies $X \cup Y \in m(U, A, F)$, and so $X \vee Y=X \cup Y \in m(U, A, F)$.

(2) For $X, Y \in m(U, A, F)$, let $Z \in m(U, A, F)$ satisfying $Z \subseteq X \cap Y$ and $X \wedge Y \preceq Z$. Then from $X \wedge Y \preceq Z$, $\Phi(X \cap Y) \subseteq Z$. Since $Z \subseteq X \cap Y$, from Theorem 3.4, $\Phi(Z) \subseteq \Phi(X \cap Y)$. It implies $Z=\Phi(Z)=\Phi(X \cap Y)=$ $X \wedge Y$, and so $X \wedge Y=Z \in m(U, A, F)$.

The complete lattice $(m(U, A, F), \preceq, \wedge, \vee)$ is called $m$ concept lattice (or object oriented soft concept lattice) and simply will be denoted by $m L(U, A, F)$.

Definition 3.11. Let $m L(U, B, F)$ and $m L(U, C, G)$ be two $m$-concept lattices. $m L(U, B, F)$ is said to be finer than $m L(U, C, G)$, which is denoted by

$m L(U, B, F) \leq m L(U, C, G) \Leftrightarrow m(U, C, G) \subseteq m(U, B, F)$. If $m L(U, B, F) \leq m L(U, C, G)$ and $m L(U, C, G) \leq$ $m L(U, B, F)$, then two $m$-concept lattices are said to be isomorphic to each other, and denoted by

$$
m L(U, B, F) \cong m L(U, C, G) \text {. }
$$

Theorem 3.12. Let $m L(U, A, F)$ be an $m$-concept lattice. Then for $C \subseteq A, m L(U, A, F) \leq m L\left(U, C, F_{C}\right)$.
Proof. From Theorem 3.7, we know that $m\left(U, C, F_{C}\right) \subseteq$ $m(U, A, F)$. So, we have $m L(U, A, F) \leq m L\left(U, C, F_{C}\right)$.

Theorem 3.13. Let $(U, A, F)$ be a soft context and $C \subseteq A$. Then $m L(U, A, F) \cong m L\left(U, C, F_{C}\right)$ if and only if $\operatorname{Im}(\mathbb{F})=$ $\operatorname{Im}\left(\mathbb{F}_{C}\right)$.

Proof. By Theorem 3.5, $\operatorname{Im}(\mathbb{F})=\operatorname{Im}\left(\mathbb{F}_{C}\right)$ if and only if $m(U, A, F)=m\left(U, C, F_{C}\right)$ if and only if $m L(U, A, F) \cong$ $m L\left(U, C, F_{C}\right)$. So, the theorem is obtained.

Example 3.14. As in Example 3.9, let us consider a soft context $(U, A, F)$. For a subset $C=\{a, b, c\}$ of $A,\left(U, C, F_{C}\right)$ is a soft context. Then we easily find that $m\left(U, C, F_{C}\right)=$ $\{\emptyset,\{1,2\},\{1,3\},\{2,5\},\{1,2,3\},\{1,2,5\},\{1,2,3,5\}, U\}$. So, $m(U, A, F)=m\left(U, C, F_{C}\right)$ and $\operatorname{Im}(\mathbb{F})=\operatorname{Im}\left(\mathbb{F}_{C}\right)$. Consequently, $m L(U, A, F) \cong m L\left(U, C, F_{C}\right)$. The following diagrams are induced by $A$ and $C \subseteq A$, respectively.

$$
\begin{aligned}
& A=\{a, b, c, d, e\} \\
& U \\
& \uparrow \\
& \{1,2,3,5\} \\
& \begin{aligned}
\{1,2,5\} & \nwarrow 1,2,3\} \\
\uparrow & \nwarrow
\end{aligned} \\
& \{2,5\}\{1,2\} \quad\{1,3\} \\
& \nwarrow \uparrow \nearrow \\
& \emptyset
\end{aligned}
$$

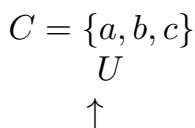

$$
\begin{aligned}
& \{1,2,3,5\} \\
& \nearrow \nwarrow \\
& \{1,2,5\} \quad\{1,2,3\} \\
& \uparrow \nwarrow \nearrow \uparrow \\
& \{2,5\} \quad\{1,2\} \quad\{1,3\} \\
& \nwarrow \uparrow \nearrow \\
& \emptyset \\
& m L(U, A, F) \\
& \cong \\
& m L\left(U, C, F_{C}\right)
\end{aligned}
$$

\section{CONCLUSION}

We showed that the set of all $m$-concepts of a given $m$-context together with the order relation between two $m$-concepts is a complete lattice, and found what is the condition for the isomorphic relation between two $m$-concept lattices. In the next research, we will study the relationships between $m$-concept lattices and formal concept lattices.

\section{ACKNOWLEDGMENTS}

This research was supported by Basic Science Research Program through the National Research Foundation of Korea(NRF) funded by the Ministry of Education (No. NRF2017R1D1A1B03031399).

\section{REFERENCES}

[1] M. I. Ali, F. Feng, X. Y. Liu, W. K. Min, M. Shabir, On some new operations in soft set theory, Computers and Mathematics with Applications, 57, 2009, 1547-1553.

[2] B. Ganter, R. Wille, Formal Concept Analysis: Mathematical Foundations, Springer, Berlin, 1999.

[3] J. Jin, K. Qin, Z. Pei, Reduction-based approaches towards constructing Galois (concept) lattices, Lecture Notes in Artificial Intelligence, 4062, Springer, Berlin, 2006, 107-113.

[4] P. K. Maji, R. Biswas, A. R. Roy, On soft set theory, Comput. Math. Appl., 45, 2003, 555-562. 
International Journal of Engineering Research and Technology. ISSN 0974-3154 Vol.13, No.3 (2020), pp. 548-551

(c) International Research Publication House. https://dx.doi.org/10.37624/IJERT/13.3.2020.548-551

[5] Min W. K., Soft sets over a common topological universe, Journal of Intelligent and Fuzzy Systems, 26(5), 2014, 2099-2106.

[6] W. K. Min, Y. K. Kim, Soft concept lattice for formal concept analysis based on soft sets: Theoretical foundations and Applications, Soft Computing, 23(19), 2019, 9657-9668. https://doi.org/10.1007/s00500-018-3532-z

[7] W. K. Min, Y. K. Kim, On Object-oriented Concepts in a Soft Context Defined by a Soft Set, International Journal of Engineering Research and Technology, 12(11), 2019, 1914-1918.
[8] D. Molodtsov, Soft set theory first results, Computers and Mathematics with Applications, 37, 1999, 19-31.

[9] R. Wille, Concept lattices and conceptual knowledge systems, Computers Mathematics with Applications, 23(6-9), 1992, 493-515.

[10] R. Wille, Restructuring the lattice theory: an approach based on hierarchies of concepts, in: I. Rival (Ed.), Ordered Sets, Reidel, Dordrecht, Boston, 1982, 445-470.

[11] Y. Y. Yao, A comparative study of formal concept analysis and rough set theory in data analysis, RSCTC 2004: Rough Sets and Current Trends in Computing, 2004, 59-68. 